\title{
On the Energetics of a Damped Beam-Like Equation for Different Boundary Conditions
}

\author{
SAJAD HUSSAIN SANDILO*, ABDUL HANAN SHEIKH*, ABDUL REHMAN SOOMRO*, AND \\ SYED FEROZ SHAH**
}

RECEIVED ON 27.07.2016 ACCEPTED ON 22.11.2016

\begin{abstract}
In this paper, the energy estimates for a damped linear homogeneous beam-like equation will be considered. The energy estimates will be studied for different BCs (Boundary Conditions) for the axially moving continuum. The problem has physical and engineering application. The applications are mostly occurring in models of conveyor belts and band-saw blades. The research study is focused on the Dirichlet, the Neumann and the Robin type of BCs. From physical point of view, the considered mathematical model expounds the transversal vibrations of a moving belt system or moving band-saw blade. It is assumed that a viscous damping parameter and the horizontal velocity are positive and constant. It will be shown in this paper that change in geometry or the physics of the boundaries can affect the stability properties of the system in general and stability depends on the axial direction of the motion. In all cases of the BCs, it will be shown that there is energy decay due to viscous damping parameter and it will also be shown that in some cases there is no conclusion whether the beam energy decreases or increases. The detailed physical interpretation of all terms and expressions is provided and studied in detail.
\end{abstract}

Key Words: Energy Estimate, Conveyor Belt System, Beam-Like, Axially Translating, Viscous Damping.

\section{INTRODUCTION}

tructural and mechanical systems are generally appearing in the class of the vibratory systems. Axially transporting elastic systems are one of them. Axially transporting systems have received a great importance and attention since last few decades. These systems are found in many physical and practical situations. Such systems may experience the energy dissipation which is known as damping [1-3]. Axially moving mechanical systems such as conveyor belts [46]; band-saw blades, magnetic and paper tapes, data storage devices, elevator cables [5], crane and mine hoisting cables, tram and train ways, pipes transporting liquids and gases, and all such type of systems are subject to vibration due to many causes.

The study of axially moving systems with damping has received great importance in design and manufacture. As vibrations cause severe damages to physical systems and structures, it is necessary to study how to reduce unnecessary noise and the vibration from these systems.

* Department of Mathematics \& Statistics, Quaid-e-Awam University of Engineering, Science \& Technology, Nawabshah.

** Department of Basic Sciences \& Related Studies, Mehran University of Engineering \& Technology, Jamshoro.

Mehran University Research Journal of Engineering \& Technology, Volume 36, No. 2, April, 2017 [p-ISSN: 0254-7821, e-ISSN: 2413-7219] 
The severe vibrations can even cause construction failures. The Tacoma Narrows suspension bridge in Washington DC, USA is good example of huge collapse where the bridge lost its stability and collapsed. Instead of such collapses; vibrations cause human uneasiness. As vibrations can create anxiety to humans therefore, it is necessary to study the methods and procedures to mitigate vibrations from the physical, structural and mechanical systems.

Damping devices are widely used to control the vibration and noise in the mechanical systems. Motion of axially transporting systems be modeled by formulations such as linear (nonlinear) string-like or linear (nonlinear) beamlike equations. Applied mathematicians, civil and mechanical engineers and physicists are continuously trying to construct mathematical models which can be directly associated to the physical systems where damping can have strong significance in reduction of unnecessary vibratory energy.

In most cases the dampers are used at boundaries to control the vibration through boundary supports [4-7] and in some other cases damping is introduced along the whole domain of the system [8]. It is possible to control the oscillation amplitudes of the 2D (Two Dimensional) plates by using dampers [7]. Akkaya and van Horssen [9] have studied the reflection and the damping properties for a wave equation and provided interesting results in case of semi-infinite string. Zhu and $\mathrm{Ni}[10]$ have provided detailed studies of energetics for an elevator cable system for different BCs. Wickert and Mote [11] have studied energetics for axially moving continua for fixed supports in case of the string and simply supports in case of the beam, but authors did not provide detailed description of energetics for different BCs and the stability criteria. Sometimes dampers can also be connected at the middle of the string or beam [12]. But the position of the damper plays significant role. If damper is attached at right position, it will damp the system and reduce the unnecessary vibration and noise, on the other hand if it is placed at wrong geometric position it may increase the vibratory energy and make the system even more unstable [13]. Chen and $\mathrm{Zu}$ [14] have done interesting work by extending the known energy and conserved functional from linear vibration to nonlinear vibration for axially moving materials.

In this paper, a damped beam-like equation which is a fourth order linear homogeneous partial differential equation has been considered where ten different $\mathrm{BCs}$ are taken into account. These BCs are studied in detail and, stability and instability of the axially moving system are provided in systematic manner for all of these BCs. The geometric structure of the pulley-support plays an important role in stabilizing or destabilizing the mechanical system. The paper is organized as follows. In Section 2, the mathematical model and the energy estimates for this model are studied in detail for all kinds of boundary conditions. In Section 3 the results and discussion is provided.

\section{THE MATHEMATICAL MODEL AND THE ENERGY ESTIMATES}

In this section, the mathematical model of the damped beam-like equation related to axially translating elastic system will be considered. The mathematical equations are obtained by the application of Hamilton's principle [8]. Following non-dimensional form of the equations are taken into account:

$\xi_{\tau \tau}+2 V \xi_{\eta \tau}+\dot{V}_{\eta}+V^{2} \xi_{\eta \eta}-\xi_{\eta \eta}+\mu \xi_{\eta \eta \eta \eta}+\delta\left(\xi_{\tau}+V \xi_{\eta}\right)=0 \quad 0<\eta<1, \tau>0 \quad(1)$ 
with the following associated $\mathrm{BCs}$,

$$
\begin{aligned}
& \xi(0 ; \tau)=\xi_{\eta}(0 ; \tau)=0, \xi(1 ; \tau)=\xi_{\eta}(1 ; \tau)=0 ; \\
& \xi(0 ; \tau)=\xi_{\eta \eta}^{\eta}(0 ; \tau)=0, \xi(1 ; \tau)=\xi_{\eta \eta}(1 ; \tau)=0 ; \\
& \xi(0 ; \tau)=\xi_{\eta}(0 ; \tau)=0, \xi(1 ; \tau)=\xi_{\eta \eta}(1 ; \tau)=0 \text {; } \\
& \xi(0 ; \tau)=\xi_{\eta \eta}(0 ; \tau)=0, \xi(1 ; \tau)=\xi_{\eta}(1 ; \tau)=0 ; \\
& \xi(0 ; \tau)=\xi_{\eta}(0 ; \tau)=0, \xi(1 ; \tau)=\xi_{\eta \eta}(1 ; \tau)=\xi_{\eta \eta \eta}(1 ; \tau)=0 ; \\
& \xi_{\eta \eta}(0 ; \tau)=\xi_{\eta \eta \eta}^{\eta}(0 ; \tau)=0, \xi(1 ; \tau)=\xi_{\eta}(1 ; \tau)=0 ; \\
& \xi(0 ; \tau)=\xi_{\eta}(0 ; \tau)=0, \xi_{\eta \eta}(1 ; \tau)=\xi_{\eta \eta \eta}(1 ; \tau)- \\
& \xi_{\eta}(1 ; \tau)-\mathrm{k} \xi(1 ; \tau)-\mathrm{V}\left(\xi_{\tau}(1 ; \tau)+\mathrm{V} \xi_{\eta}(1 ; \tau)\right)=0 ; \\
& \xi(0 ; \tau)=\xi_{\eta \eta}(0 ; \tau)=0, \xi_{\eta \eta}(1 ; \tau)=\xi_{\eta \eta \eta}(1 ; \tau)-\xi_{\eta}(1 ; \tau)-\xi_{\eta}(1 ; \tau)- \\
& \mathrm{k} \xi(1 ; \tau)-\mathrm{V}\left(\xi_{\tau}(1 ; \tau)+\mathrm{V} \xi_{\eta}(1 ; \tau)\right)=0 \text {; } \\
& \xi_{\eta \eta}(0 ; \tau)=\xi_{\eta \eta \eta}(0 ; \tau)-\xi_{\eta}(0 ; \tau)-\mathrm{k} \xi(0 ; \tau)-\mathrm{V}\left(\xi_{\tau}\left(0 ; \xi_{\tau}\right)+\right. \\
& \mathrm{V}(0 ; \tau)=0, \xi(1 ; \tau)=\xi_{\eta}(1 ; \tau)=0 \\
& \xi_{\eta \eta}(0 ; \tau)=\xi_{\eta \eta \eta}(0 ; \tau)-\xi_{\eta}(0 ; \tau)-\mathrm{k} \xi(0 ; \tau)-\mathrm{V}\left(\xi_{\tau}\left(0 ; \xi_{\tau}\right)+\right. \\
& \mathrm{V}\left(\xi_{\tau}(0 ; \tau)=\mathrm{V} \xi_{\eta}(0 ; \tau)\right)=0, \xi(1 ; \tau)=\xi_{\eta \eta}(1, \tau)=0 \text {; }
\end{aligned}
$$

where in Equations (1-2) $\xi$ models the vertical motion from equilibrium position which depends on $\eta$ and $\tau$, and where ä is related to external viscous damping coefficient, $\mu$ is related to bending stiffness and where $k$ is related to spring coefficient which is positive and constant. The term $\xi_{\eta \eta}$ corresponds to elastic force arising from horizontal tension and slopes, and the term $\xi_{\eta \eta \eta \eta}$ is related to shear force.

Now by multiplying the material velocity $\left(\xi_{\tau}+\mathrm{V} \xi_{\eta}\right)$ with Equation (1) and performing long but elementary calculations, we arrive at:

$\left.\left(\frac{1}{2}\left(\xi_{\tau}+V \xi_{\eta}\right)^{2}+\frac{1}{2} \xi_{\eta_{m}}^{2}+\frac{1}{2} \mu \xi_{\eta_{m}}^{2}\right)+\left(\left(\frac{1}{2} V\left(\xi_{\tau}+V \xi_{\eta}\right)^{2}-\xi_{\eta}\left(\xi_{\tau}+\frac{1}{2} V \xi_{\xi_{\eta}}\right)+\mu \xi_{\eta \eta} \eta_{\eta}\left(y_{\tau}+V \xi_{\eta}\right)-\mu \xi_{\eta \eta}\right)\right)\right)_{\eta}=-\delta\left(\xi_{\tau}+V \xi_{\eta}\right)^{2}$

Sum of energies (KE and PE) of a particle located at a spatial coordinate $c ̧$ at time coordinate $\tau$ is given by:

$$
\hat{E}(\tau)=\frac{1}{2}\left(\xi_{\tau}+V \xi_{\eta}\right)^{2}+\frac{1}{2} \xi_{\eta}^{2}+\frac{1}{2} \mu \xi_{\eta \eta}^{2}
$$

From Equations (3-4), it follows that:

$$
\left.\frac{\partial \hat{E}(\tau)}{\partial \tau}=-\delta\left(\xi_{\tau}+V \xi_{\eta}\right)^{2}-\left(\frac{1}{2} V\left(\xi_{\tau}+V \xi_{\eta}\right)^{2}-\xi_{\eta}\left(y_{\tau}+\frac{1}{2} V \xi_{\eta}\right)+\mu \xi_{\eta \eta}\left(\xi_{\tau}+V \xi_{\eta}\right)-\mu \xi_{\eta}\left(\xi_{\eta \tau}+\frac{1}{2} V \xi_{\eta \eta}\right)\right)\right)_{\eta}
$$

And so, energy $\mathrm{E}(\tau)$ within $(0,1)$ of the moving beam is:

$$
E(\tau)=\int_{0}^{1} \widehat{E}(\tau) d \eta
$$

By Reynold's transport theorem the time rate of change of total mechanical energy within $(0,1)$ is given as follows:

$\frac{d E}{d \tau}=\int_{0}^{1} \frac{\widehat{E}(\tau)}{\partial \tau} d \eta+\left.V \widehat{E}(\tau)\right|_{0} ^{1}$

where the term $\int_{0}^{1} \frac{\hat{E}(\tau)}{\partial \tau} d \eta$ in Equation (7) represents the local time rate of change of energy and the term $\left.V \bar{E}(\tau)\right|_{0} ^{1}$ represents the energy flux at the boundaries.

Thus from Equation (7), it follows that:

$$
\begin{aligned}
& \frac{d E}{d \tau}=-\delta \int_{0}^{1}\left(\xi_{\tau}+V \xi_{\eta}\right)^{2} d \eta-\left(\frac{1}{2} V\left(\xi_{\tau}+V \xi_{\eta}\right)^{2}-\xi_{\eta}\left(\xi_{\tau}+\frac{1}{2} V \xi_{\eta}\right)\right. \\
& \left.\left(+\mu \xi_{\eta \eta \eta}\left(\xi_{\tau}+V \xi_{\eta}\right)-\mu \xi_{\eta \eta}\left(\xi_{\eta \tau}+\frac{1}{2} V \xi_{\eta \eta}\right)\right)\right|_{0} ^{1}+\left(\frac{1}{2} V\left(\xi_{\tau}+V \xi_{\eta}\right)^{2}\right. \\
& \left.+\frac{1}{2} V\left(\xi_{\tau}+V \xi_{\eta}\right)^{2}+\frac{1}{2} V \xi_{\eta}^{2}+\frac{1}{2} \mu V \xi_{\eta \mu}^{2}\right)\left.\right|_{0} ^{1}
\end{aligned}
$$

After simplification the Equation (8) finally reduces to:

$$
\frac{d E}{d \tau}=-\delta \int_{0}^{1}\left(\xi_{\tau}+V \xi_{\eta}\right)^{2} d \eta+\left(\xi_{\eta}-\mu \xi_{\eta \eta \mu}\right)\left(\xi_{\tau}-V \xi_{\eta}\right){ }_{0}^{1} \mu \xi_{\eta \eta}\left(\xi_{\eta \tau}+V \xi_{\eta \eta}\right){ }_{0}^{1}
$$

From mechanical point of view, $-\delta \int_{0}^{1}\left(\xi_{\tau}+V \xi_{\eta}\right)^{2} d \eta$ represents the influence of viscous damping throughout the domain $(0,1)$ which obviously reduces the energy of motion, the second term represents the work done by shear and elastic forces at the boundaries $\eta=0$ and $\eta=1$ through transversal velocity, and the third term is arising due to work of bending force through slopes of the transversal velocity.

Now ten different cases of the associated BCs will be studied in detail. These BCs are very important from physical and geometrical point of view.

$$
\begin{aligned}
& \xi(0 ; \tau)=\xi_{\eta}(0 ; \tau)=0 ; \text { and } \xi(1 ; \tau)=\xi_{\eta}(1 ; \tau)=0 ;(\text { both } \\
& \text { pulleys of the beam are held fixed) }
\end{aligned}
$$

In this case at $\eta=0$ or $\eta=1$, if $\xi \Rightarrow 0 \xi_{\tau}+V \xi_{\eta}=0$ and similarity $\xi_{\eta}=0 \Rightarrow \xi_{\eta \tau}=0$, but $\xi_{\eta \eta}$ “" 0 . Thus, from Equation (9) it follows that: 
$\frac{d E}{d \tau}=-\delta \int_{0}^{1}\left(\xi_{\tau}+V \xi_{\eta}\right)^{2} d x+\left.\xi_{\eta}\left(V \xi_{\eta}\right)\right|^{1}+\left.\mu \xi_{\eta \eta}\left(V \xi_{\eta \eta}\right)\right|_{0} ^{1}$

(ii) $\quad \xi(0 ; \tau)=\xi_{\eta \eta}(0 ; \tau)=0$; and $\xi(1 ; \tau)=\xi_{\eta \eta}(1 ; \tau)=0 ;$ (both pulleys of the beam are simply supported)

In this case of simple supports at $\eta=0$; or $\eta=1$, if $\xi=0 \Rightarrow$ $\xi_{\tau}=0$, but $\xi_{\eta}$ “" 0 . Thus, from Equation (9) it follows that:

$\frac{d E}{d \tau}=-\delta \int_{0}^{1}\left(\xi_{\tau}+V \xi_{\eta}\right)^{2} d \eta+\left.\left(\xi_{\eta}+\mu \xi_{\eta \eta}\right)\left(V \xi_{\eta}\right)\right|_{0} ^{1}$

(iii) $\quad \xi(0 ; \tau)=\xi_{\eta}(0 ; \tau)=0$; and $\xi(1 ; \tau)=\xi_{\eta \eta}(1 ; \tau)=0$; (fixed support-simple support)

In this case, from Equation (9) it follows that:

$\frac{d E}{d \tau}=-\delta \int_{0}^{1}\left(\xi_{\tau}+V \xi_{\eta}\right)^{2} d \eta+\left.\left(\xi_{\eta}-\mu \xi_{\eta \eta \eta}\right)\left(\xi_{\tau}+V \xi_{\eta}\right)\right|_{0} ^{1}-\left.\mu \xi_{\eta \eta}\left(V \xi_{\eta \eta}\right)\right|^{0}$

(iv) $\quad \xi(0 ; \tau)=\xi_{\eta \eta}(0 ; \tau)=0 ;$ and $\xi(1 ; \tau)=\xi_{\eta}(1 ; \tau)=0 ;($ simple support-fixed support)

In this case of BCs it follows from Equation (9) that:

$\frac{d E}{d \tau}=-\delta \int_{0}^{1}\left(\xi_{\tau}+V \xi_{\eta}\right)^{2} d \eta-\left.\left(\xi_{\eta}-\mu \xi_{\eta \eta \eta}\right)\left(\xi_{\tau}+V \xi_{\eta}\right)\right|^{0}+\left.\mu \xi_{\eta \eta}\left(V \xi_{\eta \eta}\right)\right|^{1}$

(v)

$\xi(0 ; \tau)=\xi_{\eta}(0 ; \tau)=0 ;$ and $\xi_{\eta \eta}(1 ; \tau)=\xi_{\eta \eta \eta}(1 ; \tau)=0 ;$ (fixed support-free support)

In this case of BCs it follows from Equation (9) that:

$\frac{d E}{d \tau}=-\delta \int_{0}^{1}\left(\xi_{\tau}+V \xi_{\eta}\right)^{2} d \eta+\left.\xi_{\eta}\left(\xi_{\tau}-\mu \xi_{\eta}\right)\right|^{1}-\left.\xi_{\eta \eta}\left(V \xi_{\eta \eta}\right)\right|^{0}$

$$
\xi_{\eta \eta}(0 ; \tau)=\xi_{\eta \eta \eta}(0 ; \tau)=0 ; \text { and } \xi(1 ; \tau)=\xi_{\eta}(1 ; \tau)=0 \text {; }
$$
(free support-fixed support)

In this case of BCs it follows from Equation (9) that:

$$
\frac{d E}{d \tau}=-\delta \int_{0}^{1}\left(\xi_{\tau}+V \xi_{\eta}\right)^{2} d \eta-\left.\xi_{\eta}\left(\xi_{\tau}+V \xi_{\eta}\right)\right|^{0}+\left.\mu \xi_{\eta \eta}\left(V \xi_{\eta \eta}\right)\right|^{1}
$$

$$
\xi(0 ; \tau)=\xi_{\eta}(0 ; \tau)=0 ; \text { and } \xi_{\eta \eta}(1 ; \tau)=\mu \xi_{\eta \eta \eta}(1 ; \tau)-\xi_{\eta}
$$
$(1 ; \tau)-\mathrm{k} \xi(1 ; \tau)-\mathrm{V}\left(\xi_{\tau}(1 ; \tau)+\mathrm{V} \xi_{\eta}(1 ; \tau)\right)=0 ;$ fixed support-elastic support)
In this case of BCs it follows from Equation (9) that:

$\frac{d E}{d \tau}=-\delta \int_{0}^{1}\left(\xi_{\tau}+V \xi_{\eta}\right)^{2} d \eta-\left.k \xi\left(\xi_{\tau}+V \xi_{\eta}\right)\right|^{1}-\left.V\left(\xi_{\tau}+V \xi_{\eta}\right)^{2}\right|^{1}-\left.\mu \xi_{\eta \eta}\left(V \xi_{\eta \eta}\right)\right|^{0}$

$$
\begin{aligned}
& \xi(0 ; \tau)=\xi_{\eta \eta}(0 ; \tau)=0, \xi_{\eta \eta}(1 ; \tau)=\mu \xi_{\eta \eta \eta}(1 ; \tau)-\xi_{\eta}(1 ; \tau)- \\
& \mathrm{k} \xi(1 ; \tau)-\mathrm{V}\left(\xi_{\tau}(1 ; \tau)+\mathrm{V} \xi_{\eta}(1 ; \tau)\right)=0 ;(\text { simple support- } \\
& \text { elastic support) }
\end{aligned}
$$

In this, from Equation (9) it follows that:

$$
\left.\frac{d E}{d \tau}=-\delta \int_{0}^{1}\left(\xi_{\tau}+V \xi_{\eta}\right)^{2} d \eta-k \xi\left(\xi_{\tau}+V \xi_{\eta}\right)\right)^{1}-\left.V\left(\xi_{\tau}+V \xi_{\eta}\right)^{2}\right|^{1}-\left(\xi_{\eta}-\mu \xi_{\eta \eta \eta}\right)\left(V \xi_{\eta}\right)^{0}
$$

(ix)

$$
\begin{aligned}
& \xi_{\eta \eta}(0 ; \tau)=\mu \xi_{\eta \eta \eta}(0 ; \tau)-\xi_{\eta}(0 ; \tau)-\mathrm{k} \xi(0 ; \tau)-\mathrm{V}\left(\xi_{\tau}(0 ; \tau)+\right. \\
& \left.\mathrm{V} \xi_{\eta}(0 ; \tau)\right)=0, \xi(1 ; \tau)=\xi_{\eta}(1 ; \tau)=0, \text { (elastic support- } \\
& \text { fixed support) }
\end{aligned}
$$

In this case, from Equation (9) it follows that:

$$
\frac{d E}{d \tau}=-\delta \int_{0}^{1}\left(\xi_{\tau}+V \xi_{\eta}\right)^{2} d \eta+\left.k \xi\left(\xi_{\tau}+V \xi_{\eta}\right)\right|^{0}+\left.V\left(\xi_{\tau}+V \xi_{\eta}\right)^{2}\right|^{0}+\left.\mu \xi_{\eta \eta}\left(V \xi_{\eta \eta}\right)\right|^{1}
$$

(x)

$$
\begin{aligned}
& \xi_{\eta \eta}(0 ; \tau)=\mu \xi_{\eta \eta \eta}(0 ; \tau)-\xi_{\eta}(0 ; \tau)-\mathrm{k} \xi(0 ; \tau)-\mathrm{V}\left(\xi_{\tau}(0 ; \tau)+\right. \\
& \left.\mathrm{V} \xi_{\eta}(0 ; \tau)\right)=0, \xi(1 ; \tau)=\xi_{\eta \eta}(1 ; \tau)=0 \text {, (elastic support- } \\
& \text { simple support) }
\end{aligned}
$$

In this case, from Equation (9) it follows that:

$$
\frac{d E}{d \tau}=-\delta \int_{0}^{1}\left(\xi_{\tau}+V \xi_{\eta}\right)^{2} d \eta+\left.\left(\xi_{\eta}-\mu \xi_{\eta \eta}\right)\left(V \xi_{\eta}\right)\right|^{1}+\left.k \xi\left(\xi_{\tau}+V \xi_{\eta}\right)\right|^{0}+\left.V\left(\xi_{\tau}+V \xi_{\eta}\right)^{2}\right|^{0}
$$

\section{RESULTS AND DISCUSSION}

In this section, detailed discussion about the results obtained in previous section will be provided and their physical interpretation will be highlighted. In Equations (10-19), the temporal variation of the total mechanical energy of a translating beam equals the net rate of work done on the beam. The expressions in Equation (10-19) have simple physical interpretations. In all expressions of temporal variation of energy, the vibratory energy is reduced by the damping coefficient ä times the integral of the transversal velocities throughout the spatial domain $(0,1)$. For the BCs (fixed-fixed) in case (i), the second term 
represents the work done by elastic force with transversal velocity component at $\eta=1$ and third term represents the work done by bending moment with transversal component of force at both ends. For the BCs (simple-simple) in case (ii), the second term has physical meaning of work done by the shear forces with transversal velocity component at both ends. For the BCs (fixed-simple and simple-fixed) in cases (iii) and (iv), the second term is basically work done by shear force and work done by bending moment is given by third term. For the BCs (fixed-free and free-fixed) in cases (v) and (vi), interpretation is similar. In case of the elastic support the term $\mathrm{k} \xi \xi_{\tau}$ corresponds to work done by the spring force $\mathrm{k} \xi$ through the local velocity component $\xi_{\tau}$. Since $k$ is positive, but $\xi$ and $\xi_{\tau}$ can both be either positive or negative at a time, so the term $k \xi \xi_{\tau}$ will always be positive. Based on this interpretation, it is concluded that the system remains completely stable for the BCs (fixed-elastic) and (simple-elastic) in cases (vii) and (viii). It is important to note that for the BCs (elastic-fixed and elastic-simple) in cases (ix) and (x), the system is gaining the energy from elastic support instead of losing it and remains unstable. Note that for the cases (i), (iv), and (vi) the system is unstable due to increase in energy through the boundary(ies) and it cannot be concluded for the cases (ii), (iii), and (v) whether the energy of the beam is decreasing or increasing, and this occurs due to continuous energy exchange through the supports.

\section{CONCLUSION}

In this paper, an initial-boundary value problem for the stretched and tensioned beam equation under several different boundary conditions have been studied in detail. The studied problem is used as a mathematical model for describing lateral vibrations of a class of axially translating systems. The energy estimates provided are based upon the Reynold's transport theorem where the energy inflow and outflow are also considered at boundaries. Such energy inflow and outflow plays very significant role for the dynamics of the problem. It has been shown that for certain boundary conditions system is always stable and in some other cases the system is unstable. This shows how geometry of BCs of an engineering system changes the physics of the system.

\section{ACKNOWLEDGEMENTS}

The authors would like to thank Department of Mathematics, Quaid-e-Awam University of Engineering, Science \& Technology, Nawabshah, Pakistan, for providing logistic support to conduct this research. Authors would also like to thank unknown reviewers for their comments.

\section{REFERENCES}

[1] Darmawijoyo, and van Horssen, W.T., "On the Weakly Damped Vibrations of a String Attached to a SpringMass Dashpot System”, Journal of Vibration and Control, Volume 9, pp. 1231-1248, 2003.

[2] Darmawijoyo, and van Horssen, W.T., "On Boundary Damping for a Weakly Nonlinear Wave Equation", Nonlinear Dynamics, Volume 30, pp. 179-191, 2002.

[3] Darmawijoyo, and van Horssen, W.T., "On a Rayleigh Wave Equation with Boundary Damping”, Springer, Nonlinear Dynamics, Volume 33, No. 4, pp. 399-429, 2003.

[4] Sandilo, S.H., and van Horssen, W.T., "On Boundary Damping for an Axially Moving Beam and On Variable Length Induced Oscillations of an Elevator Cable", Proceedings of 7 th European Conference on Nonlinear Dynamics, Rome, Italy, 24-29 July, 2011.

[5] Sandilo, S.H., and van Horssen, W.T., "On Boundary Damping for an Axially Moving Tensional Beam", ASME, Journal of Vibration and Acoustics, Volume 134, pp. 0110051-8, 2012.

[6] Gaiko, N.V., and van Horssen, W.T., "On the Transverse, Low Frequency Vibrations of a Travelling String with Boundary Damping”, ASME, Journal of Vibration and Acoustics, Volume 137, No. 4, 2015. 
[7] Zarubinskaya, M.A., and van Horssen, W.T., "On Aspects of Boundary Damping for a Rectangular Plate", Journal of Sound and Vibration, Volume 292, pp. 844-853, 2006.

[8] Maitlo, A.A., Sandilo S.H., Sheikh, A.H., Malookani, R.A., and Qureshi, S., "On Damping Properties for an Axially Translating String", Science International, (Lahore), Volume 28, No. 4, pp. 3721-3727, 2016. Akkaya T., and van Horssen, W.T., "Reflection and Damping Properties for Semi-Infinite String Equations with Non-Classical Boundary Conditions", Journal of Sound and Vibration, Volume 336, pp. 179-190, 2015.

Zhu, W.D., and Ni, J., "Energetics and Stability of Translating Media with an Arbitrary Varying Length", ASME, Journal of Vibration and Acoustics, Volume 122, pp. 295-304, 2000.
Wickert, J.A., and Mote, C.D., "On the Energetics of an Axially Moving Continua", Journal of Acoustical Society of America, Volume 85, pp. 1365-1368, 1989.

[12] Main, J.A., and Jones, N.P., "Vibration of Tensioned Beams with Intermediate Damper, I: Formulation, Influence of Damper Location”, Journal of Engineering Mechanics, Volume 133, pp. 369-378, 2007.

[13] Hegedorn, P., and Seemann, W., "Modern Analytical Methods Applied to Mechanical Engineering Systems, Part-6 in Modern Methods of Analytical Mechanics and Their Applications", Rumyantsev, V.V., and Karaptyan, A.V., (Editors), CISM Courses and Lecturers No. 389, Springer-Verlage, pp. 317-328, Wien New York, 1998.

[14] Chen, L.Q., and Zu, J.W., "Energetics and Conserved Functional of Axially Moving Materials Undergoing Transverse Nonlinear Vibration", Journal of Vibration and Acoustics, Volume 126, pp. 452-455, 2004. 